\title{
Lexicographic choice functions without archimedeanicity
}

\author{
Arthur Van Camp ${ }^{1}$, Enrique Miranda ${ }^{2}$, and Gert de Cooman ${ }^{1}$
}

\begin{abstract}
We investigate the connection between choice functions and lexicographic probabilities, by means of the convexity axiom considered by [7] but without imposing any Archimedean condition. We show that lexicographic probabilities are related to a particular type of sets of desirable gambles, and investigate the properties of the coherent choice function this induces via maximality. Finally, we show that the convexity axiom is necessary but not sufficient for a coherent choice function to be the infimum of a class of lexicographic ones.
\end{abstract}

Key words: Choice functions, lexicographic probabilities, archimedeanicity, maximality

\section{Introduction}

A prominent decision model under uncertainty is that of choice functions [5]. To be able to deal with imprecise information, Seidenfeld et al. proposed an axiomatisation of coherent choice functions in [7] that generalised Rubin's [5] to allow for incomparability. They also established a representation theorem of coherent choice functions by means of probability/utility pairs.

From an imprecise probabilities perspective, choice functions can be seen as a more general model than sets of desirable gambles, because preferences are not uniquely determined by pairwise comparisons between options. We investigated this idea in [10], and in particular we studied the connections between choice functions and the notions of desirability and indifference. In order to do so, we applied the above-mentioned axiomatisation [7] to gambles

Ghent University, Data Science Lab, Technologiepark-Zwijnaarde 914, 9052 Zwijnaarde, Belgium \{Arthur.VanCamp, Gert. deCooman\}@UGent. be · University of Oviedo, Department of Statistics and Operations Research mirandaenrique@uniovi.es 
instead of horse lotteries, and also removed two axioms: (i) the Archimedean one, because it prevents choice functions from modelling the preferences captured by coherent sets of desirable gambles; and (ii) the convexity axiom, because that is incompatible with maximality as a decision rule, something that is closely tied in with coherent sets of desirable gambles. Although this alternative axiomatisation is more general, it also has the drawback of not leading to a Rubinesque representation theorem, or in other words, to a strong belief structure [2].

In the present paper, we add more detail to our previous findings [10] by investigating in more detail the implications of the convexity axiom, while still letting go of archimedeanicity. We show that, if a Rubinesque representation theorem were possible, it would involve lexicographic probabilities, but that unfortunately such a representation is not generally guaranteed. In establishing this, we derive some properties of coherent choice functions in terms of their so-called rejection sets.

The paper is organised as follows: in Section 2, we provide the basics of the theory of choice functions that we need for the rest of the paper. The connection with lexicographic probabilities and the connection with a representation theorem is addressed in Section 3. Some additional comments and remarks are provided in Section 4. Due to limitations of space, many of the proofs have been omitted.

\section{Coherent choice functions}

Consider a finite possibility space $\mathcal{X}$ in which a random variable $X$ takes values. We denote by $\mathcal{L}$ the set of all gambles - real-valued functions - on $\mathcal{X}$. Typically, a gamble $f(X)$ is interpreted as an uncertain reward: if the actual outcome turns out to be $x$ in $\mathcal{X}$, then the subject's capital changes by $f(x)$. For any two gambles $f$ and $g$, we write $f \leq g$ when $f(x) \leq g(x)$ for all $x$ in $\mathcal{X}$, and we write $f<g$ when $f \leq g$ and $f \neq g$. We collect all gambles $f$ for which $f>0$ in $\mathcal{L}_{>0}$.

For a subset $O$ of $\mathcal{L}$, we define its positive hull as posi $(O):=\left\{\sum_{k=1}^{n} \lambda_{k} f_{k}\right.$ : $\left.n \in \mathbb{N}, \lambda_{k} \in \mathbb{R}_{>0}, f_{k} \in O\right\} \subseteq \mathcal{L}$, and its convex hull as $\mathrm{CH}(O):=\left\{\sum_{k=1}^{n} \alpha_{k} f_{k}: n \in\right.$ $\left.\mathbb{N}, \alpha_{k} \in \mathbb{R}_{\geq 0}, \sum_{k=1}^{n} \alpha_{k}=1, f_{k} \in O\right\} \subseteq \mathcal{L}$, where $\mathbb{R}_{>0}\left(\mathbb{R}_{\geq 0}\right)$ is the set of all positive (non-negative) real numbers. For any two subsets $O_{1}$ and $O_{2}$ of $\mathcal{L}$ and any $\lambda$ in $\mathbb{R}$, we let $\lambda O_{1}:=\left\{\lambda f: f \in O_{1}\right\}$ and $O_{1}+O_{2}:=\left\{f+g: f \in O_{1}, g \in O_{2}\right\}$.

We denote by $\mathcal{Q}$ the set of all non-empty finite subsets of $\mathcal{L}$. Elements $O$ of $\mathcal{Q}$ are the option sets amongst which a subject can choose his preferred options.

Definition 1. A choice function $C$ is a map $C: \mathcal{Q} \rightarrow \mathcal{Q} \cup\{\varnothing\}: O \mapsto C(O)$ such that $C(O) \subseteq O$. 
The interpretation is that a choice function $C$ selects the set $C(O)$ of 'best' options in the option set $O$. Our definition resembles the one commonly used in the literature $[1,7,9]$, except for a (also not unusual) restriction to finite option sets $[6,8]$. Equivalently to a choice function $C$, we consider its rejection function $R$, defined $R(O):=O \backslash C(O)$ for all $O$ in $\mathcal{Q}$. It returns the gambles that are not selected by $C$.

In this paper, we focus on coherent choice functions.

Definition 2. We call a choice function $C$ on $\mathcal{Q}$ coherent if for all $O, O_{1}, O_{2}$ in $\mathcal{Q}, f, g$ in $\mathcal{L}$ and $\lambda$ in $\mathbb{R}_{>0}$ :

$\mathrm{C}_{1} . C(O) \neq \varnothing$

$\mathrm{C}_{2}$. if $f<g$ then $\{g\}=C(\{f, g\})$;

$\mathrm{C}_{3}$. a. if $C\left(O_{2}\right) \subseteq O_{2} \backslash O_{1}$ and $O_{1} \subseteq O_{2} \subseteq O$ then $C(O) \subseteq O \backslash O_{1}$;

b. if $C\left(O_{2}\right) \subseteq O_{1}$ and $O \subseteq O_{2} \backslash O_{1}$ then $C\left(O_{2} \backslash O\right) \subseteq O_{1}$;

$\mathrm{C}_{4}$. a. if $O_{1} \subseteq C\left(O_{2}\right)$ then $\lambda O_{1} \subseteq C\left(\lambda O_{2}\right)$;

b. if $O_{1} \subseteq C\left(O_{2}\right)$ then $O_{1}+\{f\} \subseteq C\left(O_{2}+\{f\}\right)$.

These axioms are a subset of the ones studied by Seidenfeld et al. [7], translated from horse lotteries to gambles. We have not included the Archimedean axiom, which makes our definition more general. This is important in order to make the connection with the sets of desirable gambles we recall below.

In this paper, we intend to investigate in some detail the implications of an additional axiom in [7], namely $\mathrm{C}_{5}$. if $O \subseteq O_{1} \subseteq \mathrm{CH}(O)$ then $C(O) \subseteq C\left(O_{1}\right)$ for all $O$ and $O_{1}$ in $\mathcal{Q}$, also referred to as the convexity axiom. One useful property we shall have occasion to use further on is the following:

Proposition 1. Let $C$ be a choice function on $\mathcal{L}$ satisfying $\mathrm{C}_{3} \mathrm{a}, \mathrm{C}_{4} \mathrm{a}$ and $\mathrm{C}_{5}$. Then for any $n \in \mathbb{N}, f_{1}, f_{2}, \ldots, f_{n} \in \mathcal{L}$ and $\lambda_{1}, \lambda_{2}, \ldots \lambda_{n} \in \mathbb{R}_{>0}$ :

$$
0 \in C\left(\left\{0, f_{1}, f_{2}, \ldots, f_{n}\right\}\right) \Leftrightarrow 0 \in C\left(\left\{0, \lambda_{1} f_{1}, \lambda_{2} f_{2}, \ldots, \lambda_{n} f_{n}\right\}\right) .
$$

For two choice functions $C$ and $C^{\prime}$, we call $C$ not more informative than $C^{\prime}$ - and we write $C \sqsubseteq C^{\prime}$-if $C(O) \supseteq C^{\prime}(O)$ for all $O$ in $\mathcal{Q}$. The binary relation $\sqsubseteq$ is a partial order, and for any collection $\mathcal{C}^{\prime}$ of choice functions, its infimum $\inf \mathcal{C}^{\prime}$ exists, and is given by $\inf \mathcal{C}^{\prime}(O)=\cup_{C \in \mathcal{C}^{\prime}} C(O)$ for all $O$ in $\mathcal{Q}$. Coherence is preserved under arbitrary infima [10, Proposition 3], and it is easy to show that so is convexity:

Proposition 2. For any collection $\mathcal{C}^{\prime}$ of choice functions that satisfy $\mathrm{C}_{5}$, its infimum inf $\mathcal{C}^{\prime}$ satisfies $\mathrm{C}_{5}$ as well.

One important way of defining coherent choice functions is by means of sets of desirable gambles. This connection is explored in some detail in [10]. A set of desirable gambles $D$ is simply a subset of the vector space of gambles $\mathcal{L}$. The underlying idea is that a subject finds every gamble $f$ in her set of desirable gambles strictly better than the status quo-she has a strict 
preference for the uncertain reward $f$ over 0 . As we did for choice functions, we pay special attention to coherent sets of desirable gambles, see for instance [3] for a detailed discussion.

Definition $3([3])$. A set of desirable gambles $D$ is called coherent when $D=\operatorname{posi}\left(D \cup \mathcal{L}_{>0}\right)$ and $0 \notin D$. We collect all coherent sets of desirable gambles in the set $\overline{\mathcal{D}}$.

We may associate with any $D \in \overline{\mathcal{D}}$ a strict partial order $\varangle_{D}$ on $\mathcal{L}$, by letting $f \triangleleft_{D} g \Leftrightarrow 0 \triangleleft_{D} g-f \Leftrightarrow g-f \in D$, so $D=\left\{f \in \mathcal{L}: 0 \triangleleft_{D} f\right\}$; see for instance [3]. This correspondence is one-to-one.

We may also associate with a coherent set of desirable gambles $D$ a choice function $C_{D}$ based on maximality. For any $O$ in $\mathcal{Q}$, we let $C_{D}(O)$ be the set of gambles that are undominated, or maximal, in $O$ :

$$
C_{D}(O):=\{f \in O:(\forall g \in O) g-f \notin D\}=\left\{f \in O:(\forall g \in O) f k_{D} g\right\} .
$$

Interestingly, the coherent choice function $C_{D}$ associated with a coherent set of desirable gambles $D$ need not satisfy $\mathrm{C}_{5}$ :

Proposition 3. For any coherent set of desirable gambles D, its corresponding choice function $C_{D}$ satisfies $\mathrm{C}_{5}$ if and only $\operatorname{posi}\left(D^{c}\right)=D^{c}$.

\section{Lexicographic choice functions}

Let $\overline{\mathcal{D}}_{\mathrm{L}}:=\left\{D \in \overline{\mathcal{D}}: \operatorname{posi}\left(D^{c}\right)=D^{c}\right\}$. It follows from [4, Proposition 6] that a set of gambles $D \in \overline{\mathcal{D}}_{\mathrm{L}}$ induces a linear prevision - an expectation operator with respect to a finitely additive probability - by means of the formula $P_{D}(f)$ := $\sup \{\mu \in \mathbb{R}: f-\mu \in D\}$ for all $f$ in $\mathcal{L}$. We can make an even tighter connection with the so-called lexicographic probabilities.

A lexicographic probability system is an $\ell$-tuple $p=\left(p_{1}, \ldots, p_{\ell}\right)$ of probability mass functions on $\mathcal{X}$. We associate with $p$ its expectation operator $\mathrm{E}_{p}=\left(\mathrm{E}_{p_{1}}, \ldots, \mathrm{E}_{p_{\ell}}\right)$, and its preference relation $<$ on $\mathcal{L}:$

$$
f<g \Leftrightarrow \mathrm{E}_{p}(f)<_{\mathrm{L}} \mathrm{E}_{p}(g) \text { for all } f \text { and } g \text { in } \mathcal{L},
$$

where $<_{\mathrm{L}}$ denotes the usual lexicographic order between $\ell$-tuples.

Proposition 4. Given a lexicographic probability system $\left(p_{1}, \ldots, p_{\ell}\right)$, the set of desirable gambles $D:=\{f \in \mathcal{L}: 0<f\}$ associated with the preference relation $<$ is an element of $\overline{\mathcal{D}}_{\mathrm{L}}$. Conversely, given a set of desirable gambles $D$ in $\overline{\mathcal{D}}_{\mathrm{L}}$, its associated preference relation $\varangle_{D}$ is a preference relation based on some lexicographic probability system.

Because of this result, we refer to the elements of $\overline{\mathcal{D}}_{\mathrm{L}}$ as lexicographic sets of desirable gambles, and call the elements of $\overline{\mathcal{C}}_{\mathrm{L}}:=\left\{C_{D}: D \in \overline{\mathcal{D}}_{\mathrm{L}}\right\}$ lexicographic choice functions. 
We gather from the discussion in Section 2 that the infimum of any set of lexicographic choice functions satisfies Axioms $\mathrm{C}_{1}-\mathrm{C}_{5}$. The central question that remains now, is whether any choice function that satisfies Axioms $\mathrm{C}_{1}-$ $\mathrm{C}_{5}$ is, conversely, an infimum of lexicographic choice functions. Such a representation result would make lexicographic choice functions fulfil the role of 'dually atomic' choice functions in our theory without the Archimedean axiom, in analogy with the theory with an Archimedean axiom [7], where the dually atomic choice functions are the ones induced by probability mass functions - see [2] for the terminology. In other words, we study the following:

Is, in parallel with the result in [7], every choice function $C$ that satisfies Axioms $\mathrm{C}_{1}-\mathrm{C}_{5}$ an infimum of lexicographic choice functions, or in other words, is $C(O)=\bigcup\left\{C^{\prime}(O): C^{\prime} \in \overline{\mathcal{C}}_{\mathrm{L}}, C \sqsubseteq C^{\prime}\right\}$ for all $O$ in $\mathcal{Q}$ ?

We now show that this is not the case. In our counterexample, we focus on a binary space $\mathcal{X}=\{a, b\}$. It follows from the axioms of coherence that any coherent choice function $C$ on a binary possibility space $\mathcal{X}$ can be determined by two sets: its associated set of desirable gambles $D_{C}:=\{f \in \mathcal{L}:\{f\}=$ $C\{0, f\}\}$ and a so-called rejection set $K$, which consists of the gambles $g$ in $\mathcal{L}_{\text {II }}$ and $h$ in $\mathcal{L}_{\text {IV }}$ which, taken alone, do not allow us to reject 0 , but taken together, do allow us to reject 0 :

$$
0 \in C(\{0, g\}), 0 \in C(\{0, h\}) \text {, and } 0 \in R(\{0, g, h\}) .
$$

Here $\mathcal{L}_{\mathrm{IV}}:=\{h \in \mathcal{L}: h(a)<0$ and $h(b)>0\}$ constitutes the second, and $\mathcal{L}_{\mathrm{IV}}:=\{h \in \mathcal{L}: h(a)>0$ and $h(b)<0\}$ the fourth quadrant, in the twodimensional vector space $\mathcal{L}$.

In order to construct our counterexample, consider some increasing subset $K$ of $\mathbb{R}_{>0} \times \mathbb{R}_{<0}$, and use it to define a special choice function $C_{K}$, with rejection function $R_{K}$, as follows. First of all, for any option set $O$, we let $0 \in R_{K}(\{0\} \cup O)$ if and only if

$$
O \cap \mathcal{L}_{>0} \neq \varnothing \text { or }\left(\exists \lambda_{1}, \lambda_{2} \in \mathbb{R}_{>0}\right)\left(\exists\left(\rho_{1}, \rho_{2}\right) \in K\right)\left\{\lambda_{1}\left(-1, \rho_{1}\right), \lambda_{2}\left(1, \rho_{2}\right)\right\} \subseteq O .
$$

Of course, this will define a choice function $C_{K}$ uniquely, provided that we require that $C_{K}$ should satisfy Axiom $\mathrm{C}_{4}$ b, because then, for any $O \in \mathcal{Q}$ and any $f \in O$ :

$$
f \in R_{K}(O) \Leftrightarrow 0 \in R_{K}\left(\{0\} \cup O^{\prime}\right),
$$

where $O^{\prime}:=(O-\{f\}) \backslash\{0\}$.

Proposition 5. Any choice function $C_{K}$ that is defined by Equations (1)-(2) satisfies Axioms $\mathrm{C}_{1}, \mathrm{C}_{2}, \mathrm{C}_{3} \mathrm{a}, \mathrm{C}_{4}$ a and $\mathrm{C}_{4} \mathrm{~b}$.

As far as $\mathrm{C}_{5}$ is concerned, we have established the following: 
Proposition 6. Consider any increasing $K \subseteq \mathbb{R}_{>0} \times \mathbb{R}_{<0}$. For the choice function $C_{K}$ on $\mathcal{X}=\{a, b\}$ defined by Equations (1)-(2), the following statements are equivalent:

(i) $C_{K}$ satisfies $\mathrm{C}_{5}$.

(ii) $\left(\forall\left(\kappa_{1}, \kappa_{2}\right) \in \mathbb{R}_{>0} \times \mathbb{R}_{<0}\right)\left(\kappa_{1}+\kappa_{2}>0 \Rightarrow\left(\kappa_{1}, \kappa_{2}\right) \in K\right)$.

Now, let us consider the set $K$ as depicted in the figure below. Let $C_{K}$ be

Fig. 1 The rejection set $K$ that defines the choice function $C_{K}$.

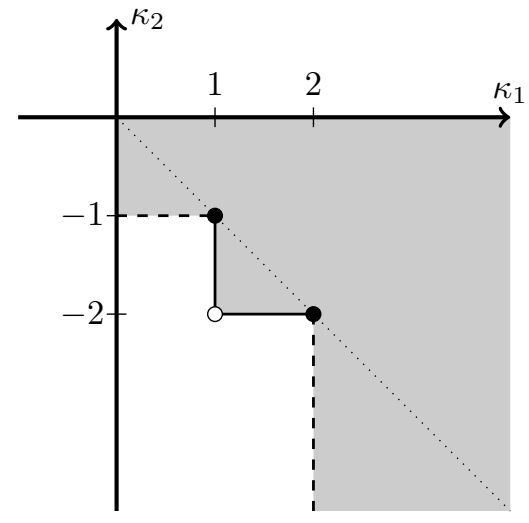

the choice function associated with this set by means of Equations (1)-(2). It follows from the discussion above that this $C_{K}$ satisfies Axioms $\mathrm{C}_{1}, \mathrm{C}_{2}$, $\mathrm{C}_{3} \mathrm{a}, \mathrm{C}_{4} \mathrm{a}, \mathrm{C}_{4} \mathrm{~b}$ and $\mathrm{C}_{5}$. Let us show that it also satisfies Axiom $\mathrm{C}_{3} \mathrm{~b}$.

Proposition 7. $C_{K}$ satisfies Axiom $\mathrm{C}_{3} \mathrm{~b}$. As a consequence, it is a coherent choice function that satisfies $\mathrm{C}_{5}$.

Proof. It can be checked that Axiom $\mathrm{C}_{3} \mathrm{~b}$ is equivalent to

$$
(\forall O \in \mathcal{Q}, \forall g \in O)\{0, g\} \subseteq R(O) \Rightarrow 0 \in R(O \backslash\{g\}) .
$$

So assume that $\{0, g\} \subseteq R_{K}(O)$. Then $g \in R_{K}(O)$ and there are $\left(\kappa_{1}, \kappa_{2}\right) \in K$ such that $\left\{\lambda_{1}\left(-1, \kappa_{1}\right), \lambda_{2}\left(1, \kappa_{2}\right)\right\} \subseteq O$ for some $\lambda_{1}$ and $\lambda_{2}$ in $\mathbb{R}_{>0}$.

If $g \neq \lambda_{1}\left(-1, \kappa_{1}\right)$ and $g \neq \lambda_{2}\left(1, \kappa_{2}\right)$ then $0 \in R_{K}(O \backslash\{g\})$ and we are done, so assume that $g=\lambda_{1}\left(-1, \kappa_{1}\right)$ or $g=\lambda_{2}\left(1, \kappa_{2}\right)$.

If $g=\lambda_{1}\left(-1, \kappa_{1}\right)$, then $0 \in R_{K}(O-\{g\})$, so there are $\left(\kappa_{1}^{\prime}, \kappa_{2}^{\prime}\right) \in K$ such that $\left\{g+\lambda_{1}^{\prime}\left(-1, \kappa_{1}^{\prime}\right), g+\lambda_{2}^{\prime}\left(1, \kappa_{2}^{\prime}\right)\right\} \subseteq O$ for some $\lambda_{1}^{\prime}$ and $\lambda_{2}^{\prime}$ in $\mathbb{R}_{>0}$, implying that $\left\{\left(-\lambda_{1}-\lambda_{1}^{\prime}, \lambda_{1} \kappa_{1}+\lambda_{1}^{\prime} \kappa_{1}^{\prime}\right),\left(-\lambda_{1}+\lambda_{2}^{\prime}, \lambda_{1} \kappa_{1}+\lambda_{2}^{\prime} \kappa_{2}^{\prime}\right)\right\} \subseteq O$.

We now have a number of possibilities for the $K$ defined in the figure above.

First of all, $\left(\frac{\lambda_{1} \kappa_{1}+\lambda_{1}^{\prime} \kappa_{1}^{\prime}}{\lambda_{1}+\lambda_{1}^{\prime}}, \kappa_{2}\right) \in K$ under any of the following conditions:

(i) $\kappa_{2}>-1$;

(ii) $\kappa_{2} \in(-2,-1]\left(\right.$ so $\left.\kappa_{1} \geq 1\right)$ and $\kappa_{1}^{\prime} \geq 1$; 
(iii) $\kappa_{2}=-2\left(\right.$ so $\left.\kappa_{1}>1\right)$ and $\kappa_{1}^{\prime} \geq 1$;

(iv) $\kappa_{2}<-2\left(\right.$ so $\left.\kappa_{1}>2\right)$ and $\kappa_{1}^{\prime} \geq 2$.

So, in any of these cases, we see that $0 \in R_{K}\left(\left\{\left(-1, \frac{\lambda_{1} \kappa_{1}+\lambda_{1}^{\prime} \kappa_{1}^{\prime}}{\lambda_{1}+\lambda_{1}^{\prime}}\right), 0,\left(1, \kappa_{2}\right)\right\}\right)$, and therefore also $0 \in R\left(\left\{g+\lambda_{1}^{\prime}\left(-1, \kappa_{1}^{\prime}\right), 0, \lambda_{2}\left(1, \kappa_{2}\right)\right\}\right)$, by Proposition 1 . Since $\lambda_{1}^{\prime}\left(-1, \kappa_{1}^{\prime}\right) \neq 0$, we infer from Axiom $\mathrm{C}_{3}$ a that indeed $0 \in R_{K}(O \backslash\{g\})$.

The remaining two possibilities are:

(v) $\kappa_{2} \leq-1\left(\right.$ so $\left.\kappa_{1} \geq 1\right)$ and $\kappa_{1}^{\prime}<1\left(\right.$ so $\left.\kappa_{2}^{\prime}>-1\right)$;

(vi) $\kappa_{2}<-2\left(\right.$ so $\left.\kappa_{1}>2\right)$ and $\kappa_{1}^{\prime} \in[1,2)\left(\right.$ so $\left.\kappa_{2}^{\prime} \geq-2\right)$.

There are now three possible cases.

If $\lambda_{1}=\lambda_{2}^{\prime}$, then $\lambda_{1} \kappa_{1}+\lambda_{2}^{\prime} \kappa_{2}^{\prime}=\lambda_{1}\left(\kappa_{1}+\kappa_{2}^{\prime}\right)>0$ and therefore also $\left(-\lambda_{1}+\lambda_{2}^{\prime}, \lambda_{1} \kappa_{1}+\lambda_{2}^{\prime} \kappa_{2}^{\prime}\right)>0$, whence $0 \in R_{K}\left(\left\{0,\left(-\lambda_{1}+\lambda_{2}^{\prime}, \lambda_{1} \kappa_{1}+\lambda_{2}^{\prime} \kappa_{2}^{\prime}\right)\right\}\right)$, by Axiom $\mathrm{C}_{2}$.

If $\lambda_{1}<\lambda_{2}^{\prime}$, then $\left(\kappa_{1}^{\prime}, \frac{\lambda_{1} \kappa_{1}+\lambda_{2}^{\prime} \kappa_{2}^{\prime}}{-\lambda_{1}+\lambda_{2}^{\prime}}\right) \in K$, and therefore also

$$
0 \in R_{K}\left(\left\{\left(-1, \kappa_{1}^{\prime}\right), 0,\left(1, \frac{\lambda_{1} \kappa_{1}+\lambda_{2}^{\prime} \kappa_{2}^{\prime}}{-\lambda_{1}+\lambda_{2}^{\prime}}\right)\right\}\right)
$$

Proposition 1 now guarantees that also

$$
0 \in R_{K}\left(\left\{\left(-\lambda_{1}^{\prime}, \lambda_{1}^{\prime} \kappa_{1}^{\prime}\right), 0,\left(-\lambda_{1}+\lambda_{2}^{\prime}, \lambda_{1} \kappa_{1}+\lambda_{2}^{\prime} \kappa_{2}^{\prime}\right)\right\}\right) .
$$

Since $\left(-\lambda_{1}^{\prime}, \lambda_{1}^{\prime} \kappa_{1}^{\prime}\right) \neq g=\left(-\lambda_{1}, \lambda_{1} \kappa_{1}\right)$-because $\kappa_{1} \geq 1$ and $\kappa_{1}^{\prime}<1$, or $\kappa_{1}>2$ and $\kappa_{1}^{\prime}<2$-, we infer from Axiom $\mathrm{C}_{3}$ a that $0 \in R_{K}(O \backslash\{g\})$.

Finally, if $\lambda_{1}>\lambda_{2}^{\prime}$, then $\left(\frac{\lambda_{1} \kappa_{1}+\lambda_{2}^{\prime} \kappa_{2}^{\prime}}{\lambda_{1}-\lambda_{2}^{\prime}}, \kappa_{2}^{\prime}\right) \in K$, implying that

$$
0 \in R_{K}\left(\left\{\left(-1, \frac{\lambda_{1} \kappa_{1}+\lambda_{2}^{\prime} \kappa_{2}^{\prime}}{\lambda_{1}-\lambda_{2}^{\prime}}\right), 0,\left(1, \kappa_{2}^{\prime}\right)\right\}\right) .
$$

Proposition 1 now guarantees that also

$$
0 \in R_{K}\left(\left\{\left(-\lambda_{1}+\lambda_{2}^{\prime}, \lambda_{1} \kappa_{1}+\lambda_{2}^{\prime} \kappa_{2}^{\prime}\right), 0,\left(\lambda_{2}^{\prime}, \lambda_{2}^{\prime} \kappa_{2}^{\prime}\right)\right\}\right) .
$$

Since $\left(-\lambda_{1}+\lambda_{2}^{\prime}, \lambda_{1} \kappa_{1}+\lambda_{2}^{\prime} \kappa_{2}^{\prime}\right) \neq g=\left(-\lambda_{1}, \lambda_{1} \kappa_{1}\right)$, because $\lambda_{2}^{\prime} \neq 0$, we infer from Axiom $\mathrm{C}_{3}$ a that indeed $0 \in R_{K}(O \backslash\{g\})$.

The proof of the case that $g=\lambda_{2}\left(1, \kappa_{2}\right)$ is similar.

To see that our $C_{K}$ is not an infimum of lexicographic choice functions, we use the following property:

Definition 4. Consider a coherent choice function $C$ and its rejection set $K$. Then $C$ is called weakly Archimedean if for all $f \in \mathcal{L}_{\mathrm{II}}$ and $g \in \mathcal{L}_{\mathrm{IV}}$ with $\operatorname{posi}(\{f, g\}) \cap \mathcal{L}_{\geq 0}=\varnothing$ :

$$
\left(\forall \epsilon \in \mathbb{R}_{>0}\right)(0 \in R(\{f+\epsilon, 0, g\}) \cap R(\{f, 0, g+\epsilon\})) \Rightarrow 0 \in R(\{f, 0, g\}) .
$$


We use this name because the property is a strictly weaker version of the Archimedean condition in [7, Axioms 3a and 3b]; it still fulfils the role of a continuity condition, but is weak enough to be still compatible with desirability, a non-Archimedean strict preference.

Proposition 8. An infimum of a non-empty set of lexicographic choice functions is weakly Archimedean.

We now see that our choice function $C_{K}$ from Proposition 7 is not an infimum of lexicographic choice functions, because it is not weakly Archimedean: note that $\{(1+\epsilon,-2),(1,-2+\epsilon)\} \subseteq K$ for all $\epsilon>0$, while $(1,-2) \notin K$.

\section{Discussion}

We have studied to which extent it is possible to have a theory of coherent choice functions that (i) as a special case allows for choosing the maximal options in the strict binary preference expressed by the notion of desirability in imprecise probabilities - meaning that we must remove the Archimedean axiom-, and that (ii) includes lexicographic probability systems as its basic building blocks. We have shown that such a theory can perfectly well incorporate the convexity axiom from [7], but that this additional axiom is not strong enough to warrant a representation theorem where every choice function is an infimum of lexicographic ones. It is still an open problem to uncover additional axioms that will guarantee such representation. We suspect that our weak archimedeanicity will play an important role in solving it.

Acknowledgements The research reported in this paper has been supported by project TIN2014-59543-P.

\section{References}

1. Aizerman M (1984) New problems in the general choice theory. Soc Ch Welf 2:235-282

2. De Cooman G (2005) Belief models: an order-theoretic investigation. Ann Math Art Intel 45:5-34

3. De Cooman G, Quaeghebeur E (2012) Exchangeability and sets of desirable gambles. Int J App Reas 53:363-395

4. Miranda E, Zaffalon M (2010) Notes on desirability and coherent lower previsions. Ann Math Art Intel 60:251-309

5. Rubin H (1987) A weak system of axioms for "rational" behavior and the nonseparability of utility from prior. Stat Risk Model 5:47-58

6. Schwartz T (1972) Rationality and the Myth of the Maximum. Noûs 6:97-117

7. Seidenfeld T, Schervisch M, Kadane J (2010) Coherent choice functions under uncertainty. Synth 172:157-176

8. Sen A (1971) Choice functions and revealed preference. Rev Econ Stud 38:307-317 
9. Sen A (1977) Social choice theory: a re-examination. Econom 45:53-89

10. Van Camp A, De Cooman G, Miranda E, Quaeghebeur E (2015) Modelling indifference with choice functions. Proc ISIPTA'15, 305-314 